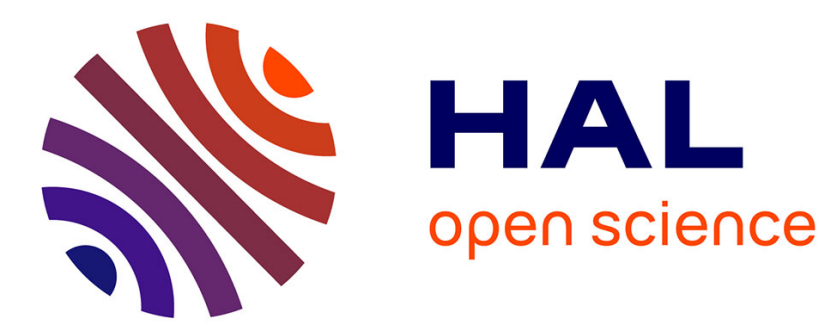

\title{
Decoupled models for vehicle dynamics and estimation of coupling terms
}

H. Nasser, N. M'Sirdi, a Naamane Lsis

\section{To cite this version:}

H. Nasser, N. M'Sirdi, a Naamane Lsis. Decoupled models for vehicle dynamics and estimation of coupling terms. 18th Mediterranean Conference on Control and Automation, MED'10, Jun 2010, Marrakech, Morocco. hal-02496392

\section{HAL Id: hal-02496392 \\ https://hal.science/hal-02496392}

Submitted on 3 Mar 2020

HAL is a multi-disciplinary open access archive for the deposit and dissemination of scientific research documents, whether they are published or not. The documents may come from teaching and research institutions in France or abroad, or from public or private research centers.
L'archive ouverte pluridisciplinaire HAL, est destinée au dépôt et à la diffusion de documents scientifiques de niveau recherche, publiés ou non, émanant des établissements d'enseignement et de recherche français ou étrangers, des laboratoires publics ou privés. 


\title{
Decoupled models for vehicle dynamics and estimation of coupling terms
}

\author{
H. NASSER, N. K. M'SIRDI, A. NAAMANE LSIS, CNRS UMR 6168
}

\begin{abstract}
This paper presents different dynamic models of vehicles to compare their dynamics. The main objective is to appreciate the couplings between the different model blocks after splitting systems to five sub models. The passivity approach is used and illustrated by simulation results obtained by the proposed simulator SimK106N.

Keywords: vehicles; dynamic models; submodels; coupling
\end{abstract} terms; simulation.

\section{INTRODUCTION :}

The intelligent vehicle is a relevant research issue for autonomous Vehicle and assistance system. This topic requires accurate models representing the dynamic vehicle behavior. A nominal dynamic vehicle model with different Degrees of Freedom (DoF) is developed by using the classical Robotics techniques. The main idea is to compare several vehicles dynamics in order to learn about the mobile behavior. This will help to emphasize of systems parts in the dynamics, like for example suspensions or chassis rotations on the behavior.

The first stage of this work consists in splitting all variable structure systems in only two coupled sub systems (M'sirdi and A. R.) [1], respectively, the frame and wheels. This decomposition is achieved to permit the analysis and control, by exploiting the passivity theory. This passivity approach allows to understand the stabilizing influences of non linear parameters present in the analytical expression of the sub-models.

Few researches approached the study and the problem of the variable structure system. There are no, in our knowledge, studies which take in to account all kinds of vehicles in the same time as a real application to analyze and simulate the model parts and theirs dynamic behavior. We are going to try throughout this paper to describe the dynamic behavior and the advancement of the coupling terms relating different components of the vehicles.

A simulator, under Matlab/Simulink (SimK106N [2]), is used for modeling and simulating different vehicle dynamics behavior in interaction with its environment. This simulation prototype has been validated with experimental results of LCPC's Peugeot 406 (M'sirdi and al. [1]). It is transformed for the simulation of our various models. This simulator is used to examine the evolution of vehicle subsystems in the case of a variable structure system. This new structure of the dynamic model is very suitable for surveillance and diagnosis purposes based on robust observers (sliding mode) and passivity based control.

\section{DYNAMIC MODEL OF THE VEHICLE:}

Our objective is to establish a model describing the dynamics of all considered vehicles for comparison (car, kart, quad, motorcycle and bike). Nevertheless, we will obtain a simulated behavior very close to the real physical system in a typical configuration of the vehicle and its environment. To compute the global system of equations of the kart model, we have used symbolic calculations software (Maple). This program allows generating the equations of the geometrical, kinematic and dynamic models of global system with rigid bodies.

\section{A. Hypotheses of modeling:}

The considered assumptions allow reducing the complexity of the system while guaranteeing certain degree of realism and efficiency of modeling. The model of dynamic behavior is generated by the method of Lagrange, assuming the vehicle in the air without contact. The wheels will undergo the constraint of the tire-ground contact and the generated force will obey geometrical and kinematic models calculated at points of tire-ground contact.

To represent the tire-ground contact, we can use the model of Pacejka (Magic formula) (H.B. Pacejka [3]). To take into account only the important dynamic influence, we propose the following assumptions (N.K. M'sirdi B.J. [4] [5]):

We consider as main mechanical bodies of the vehicle, a frame and wheels;

The frame is considered as a rigid body with $6 \mathrm{DoF}$;

Each wheel is considered rigid body in rotation around its frame;

The contact is punctual and located in the wheel symmetry plan at a distance equal to a radius $\mathrm{R}$;

The dynamics of variation of rolling radius is assumed to be negligible;

The pneumatic contact is assumed permanent.

B. Geometric model :

The tree structure is used to draw the reference marks, to raise the arborescence of the mechanism and to define the terminal organs. Figure 1 illustrates the tree structure of global vehicle. The reference R0 is arbitrary defined according to simplified assumptions. The 3D movement of the vehicle is represented by:

$\xi=\left[q_{1}, q_{2}, q_{3}, q_{4}, q_{5}, q_{6}\right]^{T}$

These six degrees of freedom are composed by 5 virtual non dynamics bodies and the frame.

- R0 is attached to the ground, R1, R2 ... R5 are the virtual bodies which define the posture of the vehicle, and their variables are $q_{1}, q_{2}, q_{3}, q_{4}$ and $q_{5}$;

- R6 is the frame, presented by the variable $q 6$.

The vector of generalized coordinates for the $\mathrm{P} 406, \mathrm{q} \in \mathrm{R}^{16}$ is defined as following:

$q=\left[q_{i}\right]^{T}$ with $\mathrm{i}=1 \ldots 16$,

With $R 11, R 21, R 31$ and $R 41$ represent the suspension system $\left(q_{7}, q_{8}, q_{9}, q_{10}\right), \mathrm{R} 12$ and R22 $\left(q_{11}, q_{12}\right)$ the steering locks around the axis $\mathrm{z}, R 13, R 32, R 23$ and $R 42$ (or $\left.q_{13}, q_{14}, q_{15}, q_{16}\right)$ are the rotations of four wheels around their axis $\mathrm{y}, \dot{q}, \ddot{q} \in \mathrm{R}^{16}$ are respectively the vectors of speeds and corresponding generalized accelerations (M'sirdi 2007 [6]). 

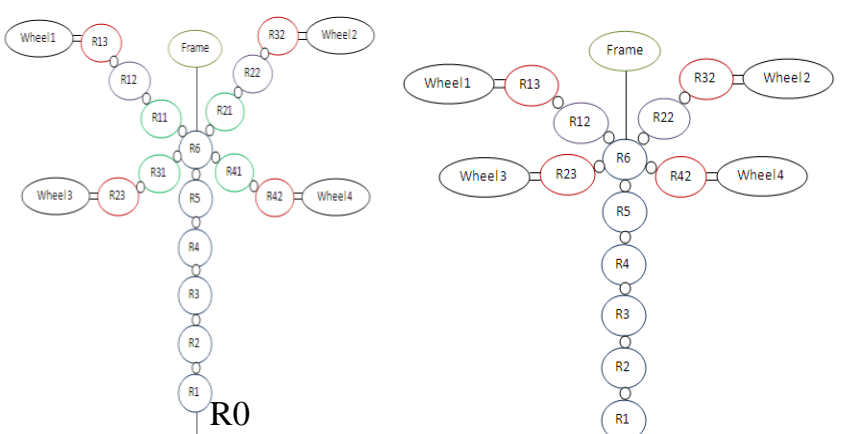

Fig. 1 \& 2: Tree representation of global vehicle (left) \& Right : of the kart
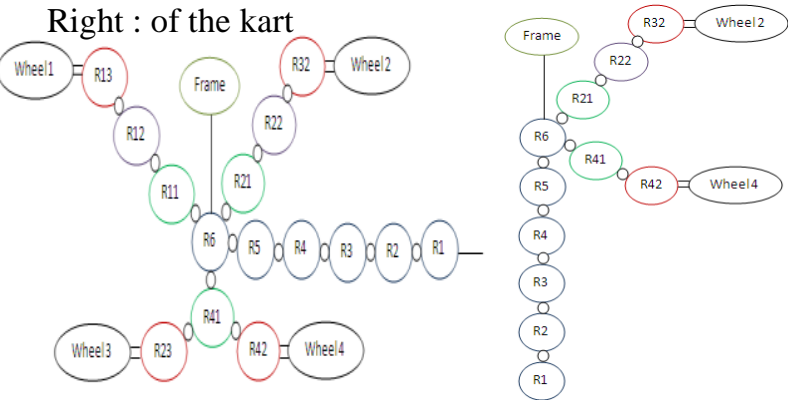

Fig. 3 left: Tree representation of the quad

Fig. 4 right: Tree representation of the motorcycle

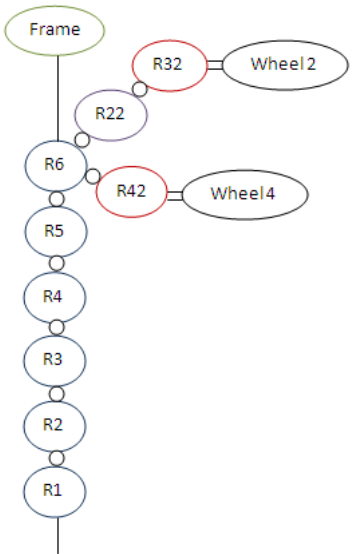

Fig. 5: Tree representation of bicycle

We can summarize the DoF belonging to each system in the following table:

\begin{tabular}{llllll}
\hline RO & P406 & QUAD & KART & MOTO & BIKE \\
\hline DoF & 16 & 15 & 12 & 11 & 9 \\
\hline
\end{tabular}

Table1: DoF for each vehicle

C. Dynamic model:

Several formalisms, in the literature, can be used to obtain dynamic models of vehicles. The most often used are:

- $\quad$ The formalism of Lagrange (Khalil [7]) (Beurier[8]);

- $\quad$ The formalism of Newton-Euler (Khalil [9]).

In this study, we are more interested in the formalism of Lagrange. This formalism describes the equations of the movement in terms of energy which is defined by the following expression:

$\Gamma_{i}=\frac{d}{d t} \frac{d L}{d \dot{q}_{i}}-\frac{d L}{d q_{i}} \mathrm{i}=1 \ldots \mathrm{n}$,

- With L : Lagrangian of the equal system in E-U ;

- $\quad$ E : Total kinetic energy of the system;

- $\quad$ U: Total potential energy of the system.

The vehicle nominal model is developed assuming that the pneumatic contact is permanent and reduced to a single point for each wheel. The dynamics of the vehicle can be described by the passive model (M'sirdi 2004): $\tau=M(q) \ddot{q}+C(q, \dot{q}) \dot{q}+V(q, \dot{q})+\eta_{0}(t, q, \dot{q})$
$\tau=\Gamma_{e}+\Gamma=\Gamma_{e}+J^{T} F \quad F=f(\lambda, \sigma, \alpha, q)$

The inertia matrix $M(q)$ of the system is defined in $\mathrm{R}^{16 \times 16}$, which is a symmetric positive definite matrix. The coefficients of the matrix $C(q, \dot{q})$ of centrifugal and Coriolis forces are established by respecting the property of passivity (M'sirdi [2]).

The vector $V(q, \dot{q})$ is composed by the gravity and frictional forces at the joints.

The vector $\eta_{0}(t, q, \dot{q})$ represents the uncertainties and the neglected dynamics in the vehicle modeling.

The equation (4) demonstrates that input torque consists of two parts; the first part is produced by the active articulations such as motorization, braking and steering. The second one represents the generalized forces due to the contact applied at the terminal organs. These forces of tireground contact (longitudinal, lateral and normal of each wheel) are gathered in the vector F.

This Kino-dynamic model, with constraints attached to the tire-ground contact, possesses the following properties:

- P1: The inertia matrix $M(q)$ is Symmetric Positive Definite (SPD) and its opposite is uniformly restricted.

- P2: With an appropriate definition of $C(q, \dot{q})$ the matrix $A(q, \dot{q})=\dot{M}(q)-2 . C(q, \dot{q})$ is Antisymmetric:

$v^{T} A(q, \dot{q}) v=v^{T}(\dot{M}(q)-2 . C(q, \dot{q})) v=0 \forall v \in R$

- P3: The dynamic equation (4) can be put in a linear parameter form in a set of dynamic parameter $\theta$ like $\tau=\varphi^{T} \theta$.

The property $\mathrm{P} 1$ assures that the effect acceleration function is inertial (stable system). P2 assures that the transfer of $\tau-\eta_{0}$ toward $\dot{q}$ is a passive system and verifies the inequality of Popov. It assures a sufficient condition of system stability linked to its passivity. These proprieties are verified in the same time as computing the model equation in Maple software.

III. DIVISION OF THE MODEL IN BLOCKS:

Our challenge is to describe the vehicle dynamics in terms of blocks and find a common area that connects the five systems studied in paragraph 2 . These models have almost the same major components, indeed we have identified five different blocks as follows:

- $\quad$ Translations of the frame (x,y and $\mathrm{z}$ );

- $\quad$ Rotations of the frame (psi, theta and phi);

Suspension System; - Steering; - Spins wheels.

Afterwards we will split each vehicle in the form of blocks and show that the coupling terms are passive and have low values. Hence the possibility of applying our observers and robust command, and switch easily between different systems.

We subdivided the vehicles into three subsets as follows:

- The global model is P406 which is the biggest model;

- Second subset contains Quad and motorcycle;

- Third subset includes kart and bike.

A. First application for a car (P406):

We split the car dynamic model while considering:

the inertia matrix $(5 \times 5)$ and the state vector are expressed as following sets (M'sirdi IROS [10]): $\quad h^{T}=\left[h_{1}^{T} h_{2}^{T} h_{3}^{T} h_{4}^{T} h_{5}^{T}\right]$ With: $h_{1}^{T}=\left[q_{1} q_{2} q_{3}\right] h_{2}^{T}=\left[q_{4} q_{5} q_{6}\right], h_{3}^{T}=$

$\left[q_{7} q_{8} q_{9} q_{10}\right], h_{3}^{T}=\left[q_{11} q_{12}\right] h_{4}^{T}=\left[q_{13} q_{14} q_{15} q_{16}\right]$

In the same way, we subdivide vectors $\Gamma_{e}, V(q, \dot{q})$ and $\eta_{0}(t, q, \dot{q})$ as it follows:

$\Gamma_{e}=\left[0,0,0, \Gamma_{e 3}, \Gamma_{e 4}\right]^{\mathrm{T}}$

$V(q, \dot{q})=\left[\begin{array}{llll}V_{1} V_{2} & V_{3} & 0 & 0\end{array}\right]^{T}$ 
$\eta_{0}(t, q, \dot{q})=\left[\eta_{1} \eta_{2} \eta_{3} \eta_{4} \eta_{5}\right]^{T}$

The 16 DoF model of the car has the following form:

$\left[\begin{array}{c}0 \\ 0 \\ 0 \\ \Gamma_{\mathrm{e} 4} \\ \Gamma_{e 5}\end{array}\right]+\left[\begin{array}{c}J_{1}^{T} \\ J_{2}^{T} \\ J_{3}^{T} \\ 0 \\ 0\end{array}\right] F=\left[\begin{array}{ccccc}M_{11} & M_{12} & M_{13} & 0 & 0 \\ M_{21} & M_{22} & M_{23} & M_{24} & M_{25} \\ M_{31} & M_{32} & M_{33} & 0 & 0 \\ 0 & M_{42} & M_{43} & M_{44} & 0 \\ 0 & M_{52} & 0 & 0 & M_{55}\end{array}\right]\left[\begin{array}{c}\ddot{h}_{1} \\ \ddot{h}_{2} \\ \ddot{h}_{3} \\ \ddot{h}_{4} \\ \ddot{h}_{5}\end{array}\right]+$

$\left[\begin{array}{ccccc}0 & C_{12} & C_{13} & 0 & 0 \\ 0 & C_{22} & C_{23} & C_{24} & C_{25} \\ 0 & C_{32} & 0 & 0 & 0 \\ 0 & C_{42} & 0 & 0 & C_{45} \\ 0 & C_{52} & 0 & C_{54} & 0\end{array}\right]\left[\begin{array}{c}\dot{h}_{1} \\ \dot{h}_{2} \\ \dot{h}_{3} \\ \dot{h}_{4} \\ \dot{h}_{5}\end{array}\right]+\left[\begin{array}{c}V_{1} \\ V_{2} \\ V_{3} \\ 0 \\ 0\end{array}\right]+\left[\begin{array}{c}\eta_{1} \\ \eta_{2} \\ \eta_{3} \\ \eta_{4} \\ \eta_{5}\end{array}\right]$

The model is described by five equations which correspond respectively to the frame translations, frame rotations, suspension system, the steering and the wheels rotations.

From this new representation, we obtain the forces translations as following:

$F_{T}=J_{1}^{T} F=M_{11} \ddot{h}_{1}+M_{12} \ddot{h}_{2}+M_{13} \ddot{h}_{3}+C_{12} \dot{h}_{2}+C_{13} \dot{h}_{3}+V_{1}+\eta_{1}$

The next equation describes the frame rotations forces.

$F_{R}=J_{2}^{T} F=M_{21} \ddot{h}_{1}+M_{22} \ddot{h}_{2}+M_{23} \ddot{h}_{3}+M_{24} \ddot{h}_{4}+$

$M_{25} \ddot{h}_{5}+C_{22} \dot{h}_{2}+C_{23} \dot{h}_{3}+C_{24} \dot{h}_{4}+C_{25} \dot{h}_{5}+V_{2}+\eta_{2}$ (a11)

The equation below represents the forces of suspension system (four dampers): (a12)

$F_{\text {susp }}=J_{3}^{T} F=M_{31} \ddot{h}_{1}+M_{32} \ddot{h}_{2}+M_{33} \ddot{h}_{3}+C_{32} \dot{h}_{2}+V_{3}+\eta_{3}$

The forces of steering and wheels rotations are: (a13)

$\Gamma_{e 4}=M_{42} \ddot{h}_{2}+M_{43} \ddot{h}_{3}+M_{44} \ddot{h}_{4}+C_{42} \dot{h}_{2}+C_{45} \dot{h}_{5}+V_{4}+\eta_{4}$

$\Gamma_{e 5}=M_{52} \ddot{h}_{2}+M_{55} \ddot{h}_{5}+C_{52} \dot{h}_{2}+C_{54} \dot{h}_{4}+V_{5}+\eta_{5} \quad$ (a14)

Most of the researchers neglect the effect of coupling terms.

The spitting of the 16 DoF model is rich in terms of data, especially, the evolution of the coupling terms $\eta_{c}^{i}$ which connect the different submodels. We can show that these variables are bounded $\forall \mathrm{t}$ and the submodels can be written: $\sum 11: \ddot{h}_{1}=f_{1}\left(h_{1}, \dot{h}_{1}, F_{T}\right)+\eta_{c}^{1}$

$\sum 12: \ddot{h}_{2}=f_{2}\left(h_{2}, \dot{h}_{2}, F_{R}\right)+\eta_{c}^{2}$

$\sum 2: \ddot{h}_{3}=f_{3}\left(h_{3}, \dot{h}_{3}, F_{\text {susp }}\right)+\eta_{c}^{3}$

$\sum 31: \ddot{h}_{4}=f_{4}\left(h_{4}, \dot{h}_{4}, \Gamma_{e 4}\right)+\eta_{c}^{4}$

$\sum 32: \ddot{h}_{5}=f_{5}\left(h_{5}, \dot{h}_{5}, \Gamma_{e 5}\right)+\eta_{c}^{5}$

A.1. Representation in 3 subsystems: - Movements of the frame: The equation (a20) gives a general frame representation (translation and rotation):

$$
\sum 1\left[\begin{array}{l}
F_{T} \\
F_{R}
\end{array}\right]=\left[\begin{array}{ll}
M_{11} & M_{12} \\
M_{21} & M_{22}
\end{array}\right]\left[\begin{array}{l}
\ddot{h}_{1} \\
\ddot{h}_{2}
\end{array}\right]+\left[\begin{array}{l}
C_{12} \\
C_{22}
\end{array}\right] \dot{h}_{2}+\left[\begin{array}{l}
V_{1} \\
V_{2}
\end{array}\right]+\left[\begin{array}{l}
\eta_{c}^{1} \\
\eta_{c}^{2}
\end{array}\right]
$$

The two coupling terms $\eta_{c}^{1}$ and $\eta_{c}^{2}$ verify:

$\eta_{c}^{1}=M_{13} \ddot{h}_{3}+C_{13} \dot{h}_{3}+\eta_{1}$

$\eta_{c}^{2}=M_{23} \ddot{h}_{3}+M_{24} \ddot{h}_{4}+M_{25} \ddot{h}_{5}+C_{23} \dot{h}_{3}+C_{24} \dot{h}_{4}+$

$C_{25} \dot{h}_{5}+\eta_{2}$

By choosing the state variables $x_{11}=\left(h_{1}, h_{2}\right)$ and $x_{12}=$ $\left(\dot{h}_{1}, \dot{h}_{2}\right)$. We have the following state representation:

$\left\{\begin{aligned} \dot{x}_{11} & =x_{12} \\ \dot{x}_{12} & =M_{1}^{-1}\left(J_{12}^{T} F-C_{1} x_{12}-V_{12}-\mu_{1}\right) \\ y_{1} & =s\left(x_{11}, x_{12}\right)\end{aligned}\right.$

With: $M_{1}=\left[\begin{array}{ll}M_{11} & M_{12} \\ M_{21} & M_{22}\end{array}\right], C_{1}=\left[\begin{array}{l}C_{12} \\ C_{22}\end{array}\right], J_{12}^{T}=\left[\begin{array}{l}J_{1}^{T} \\ J_{2}^{T}\end{array}\right], V_{12}=\left[\begin{array}{l}V_{1} \\ V_{2}\end{array}\right]$ and $\mu_{1}=\left[\begin{array}{l}\eta_{c}^{1} \\ \eta_{c}^{2}\end{array}\right]$ Which are respectively the inertia matrix, the matrix of centrifugal and Coriolis forces, the reduced Jacobian matrix, vector gravity and the vector of coupling terms linked to the first sub system.

- Suspension system:
The suspension system $\left(\sum 2\right)$ contains the fours suspensions in the car.

$F_{\text {susp }}=M_{33} \ddot{h}_{3}+V_{3}+\eta_{c}^{3}$

$\eta_{c}^{3}=M_{31} \ddot{h}_{1}+C_{32} \dot{h}_{2}+M_{32} \ddot{h}_{2}+\eta_{3}$.

Let $x_{2}=\left(x_{21}, x_{22}\right)=\left(h_{3}, \dot{h}_{3}\right)$, the state space representation of the subsystem $\left(\sum 2\right)$ is then:

$\left\{\begin{array}{l}\dot{x}_{21}=x_{22} \\ \dot{x}_{22}=M_{1}^{-1}\left(J_{3}^{T} F-V_{3}-\mu_{2}\right) \\ y_{2}=s\left(x_{21}, x_{22}\right)\end{array}\right.$
Where $\mu_{2}=\eta_{c}^{3}$

\section{- Dynamic of wheels:}

The wheels dynamic $\left(\sum 3\right)$ contains two blocks: the steering of the two front wheels $\left(\sum 31\right)$ and the rotations of four wheels following their axis $y\left(\sum 32\right)$.

The equations (a13, a14) give the following dynamic model: $\quad \Gamma_{e 4}=M_{44} \ddot{h}_{4}+C_{45} \dot{h}_{5}+\eta_{c}^{4}$

$\Gamma_{e 5}=M_{55} \ddot{h}_{5}+C_{54} \dot{h}_{4}+\eta_{c}^{5}$

By identification, we obtain the coupling terms $\eta_{c}^{4}$ and $\eta_{c}^{5}$ :

$\eta_{c}^{4}=M_{42} \ddot{h}_{2}+M_{43} \ddot{h}_{3}+C_{42} \dot{h}_{2}+\eta_{4}$

$\eta_{c}^{5}=M_{52} \ddot{h}_{2}+C_{52} \dot{h}_{2}+\eta_{5}$

If we combine the equations together we will have:

$\left[\begin{array}{l}\Gamma_{e 4} \\ \Gamma_{e 5}\end{array}\right]=\left[\begin{array}{cc}M_{44} & 0 \\ 0 & M_{55}\end{array}\right]\left[\begin{array}{l}\ddot{h}_{4} \\ \ddot{h}_{5}\end{array}\right]+\left[\begin{array}{lr}0 & C_{34} \\ C_{43} & 0\end{array}\right]\left[\begin{array}{l}\dot{h}_{4} \\ \dot{h}_{5}\end{array}\right]+\left[\begin{array}{l}\eta_{c}^{4} \\ \eta_{c}^{5}\end{array}\right]$

For the same reasoning, we chose the state variables $x_{21}=\left(h_{4}, h_{5}\right)$ and $x_{22}=\left(\dot{h}_{4}, \dot{h}_{5}\right)$, in order to obtain the following state representation :

$\left\{\begin{array}{l}\dot{x}_{21}=x_{22} \\ \dot{x}_{22}=M_{2}^{-1}\left(\Gamma_{e 45}-C_{2} x_{22}-\mu_{3}\right) \\ y_{2}=s\left(x_{21}, x_{22}\right)\end{array}\right.$

With : $M_{2}=\left[\begin{array}{cc}M_{44} & 0 \\ 0 & M_{55}\end{array}\right], C_{2}=\left[\begin{array}{lr}0 & C_{45} \\ C_{54} & 0\end{array}\right], \Gamma_{e 45}=\left[\begin{array}{l}\Gamma_{e 4} \\ \Gamma_{e 5}\end{array}\right]$, and $\mu_{3}=\left[\begin{array}{l}\eta_{c}^{4} \\ \eta_{c}^{5}\end{array}\right]$ Which are respectively the reduced inertia matrix, the reduced matrix of centrifugal and Coriolis forces, the vector of input torque and the vector of coupling terms attached to the third sub system.

\section{B. Second application for a Quad:}

Quad is an all-terrain vehicle, also known as a fourwheeler, with one seat that is straddled by the driver, along with handlebars for steering control.

It has only three dampers-springs:

- Two front dampers-springs;

Single rear damper-spring.

We split the dynamic model while considering: the inertia matrix (5x5) and the state vector are expressed as following sets: $h^{T}=\left[h_{1}^{T} h_{2}^{T} h_{3}^{T} h_{4}^{T} h_{5}^{T}\right]$

With: $h_{1}^{T}=\left[q_{1} q_{2} q_{3}\right] h_{2}^{T}=\left[q_{4} q_{5} q_{6}\right], h_{3}^{T}=\left[q_{7} q_{8} q_{9}\right], h_{3}^{T}=$

$\left[q_{10} q_{11}\right] h_{4}^{T}=\left[q_{12} q_{13} q_{14} q_{15}\right]$

In the same way, we subdivide vectors $\Gamma_{e}, V(q, \dot{q})$ and $\eta_{0}(t, q, \dot{q})$ as it follows:

$\Gamma_{e}=\left[0,0,0, \Gamma_{e 3}, \Gamma_{e 4}\right]^{\mathrm{T}}$

$V(q, \dot{q})=\left[\begin{array}{llll}V_{1} V_{2} & V_{3} & 0 & 0\end{array}\right]^{T}$

$\eta_{0}(t, q, \dot{q})=\left[\eta_{1} \eta_{2} \eta_{3} \eta_{4} \eta_{5}\right]^{T}$

Then the 15 DoF model of the quad has the following fo 


$$
\left[\begin{array}{c}
0 \\
0 \\
0 \\
\Gamma_{\mathrm{e} 4} \\
\Gamma_{e 5}
\end{array}\right]+\left[\begin{array}{c}
J_{1}^{T} \\
J_{2}^{T} \\
J_{3}^{T} \\
0 \\
J_{5}^{T}
\end{array}\right] F=\left[\begin{array}{ccccc}
M_{11} & M_{12} & M_{13} & 0 & 0 \\
M_{21} & M_{22} & M_{23} & M_{24} & M_{25} \\
M_{31} & M_{32} & M_{33} & 0 & 0 \\
0 & M_{42} & M_{43} & M_{44} & 0 \\
0 & M_{52} & 0 & 0 & M_{55}
\end{array}\right]\left[\begin{array}{l}
\ddot{h}_{1} \\
\ddot{h}_{2} \\
\ddot{h}_{3} \\
\ddot{h}_{4} \\
\ddot{h}_{5}
\end{array}\right]+
$$$$
\left[\begin{array}{ccccc}
0 & C_{12} & C_{13} & 0 & 0 \\
0 & C_{22} & C_{23} & C_{24} & C_{25} \\
0 & C_{32} & 0 & 0 & 0 \\
0 & C_{42} & 0 & 0 & C_{45} \\
0 & C_{52} & 0 & C_{54} & 0
\end{array}\right]\left[\begin{array}{c}
\dot{h}_{1} \\
\dot{h}_{2} \\
\dot{h}_{3} \\
\dot{h}_{4} \\
\dot{h}_{5}
\end{array}\right]+\left[\begin{array}{c}
V_{1} \\
V_{2} \\
V_{3} \\
0 \\
0
\end{array}\right]+\left[\begin{array}{c}
\eta_{1} \\
\eta_{2} \\
\eta_{3} \\
\eta_{4} \\
\eta_{5}
\end{array}\right]
$$

The model is described by five equations which correspond respectively to the frame translations, frame rotations, suspension system, the steering and the wheels rotations.

In conclusion, the difference with the previous car model is in the equation (b9) at the Jacobian level. The term $J_{5}^{T}$ is for description of the single back suspension axle-tree. Thus it will change the block of wheels rotations.

\section{Third application for a kart:}

The Kart which we dispose is a kind of battery-driven vehicle equipped with 4 mass OPTIMA batteries and an engine with separate excitement (brushless). The maximum speed is about $70 \mathrm{Km} / \mathrm{h}$ [H. NASSER, N.K. [11]]. It is generally accepted as the most economic form of motorsport available. Also wheels and tires are much smaller than those used on a normal car. Missing of a suspension system reduces the model of $4 \mathrm{DoF}$ compared to the 16 DoF model of Peugeot 406 (M'sirdi 2004, 2007).

We split the previous dynamic model while considering: the inertia matrix $(5 \times 5)$ and the state vector is expressed as following sets: $\quad h^{T}=\left[h_{1}^{T} h_{2}^{T} 0 h_{3}^{T} h_{4}^{T}\right]$

With: $h_{1}^{T}=\left[q_{1} q_{2} q_{3}\right] h_{2}^{T}=\left[q_{4} q_{5} q_{6}\right], h_{3}^{T}=\left[q_{7} q_{8}\right]$

$h_{4}^{T}=\left[q_{9} q_{10} q_{11} q_{12}\right]$

In the same way, we subdivide vectors $\Gamma_{e}, V(q, \dot{q})$ and $\eta_{0}(t, q, \dot{q})$ as it follows:

$\Gamma_{e}=\left[0,0,0, \Gamma_{e 4}, \Gamma_{e 5}\right]^{\mathrm{T}}$

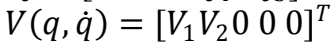

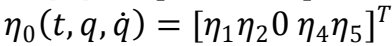

The 12 Dof model of the kart has the following form:

$$
\left[\begin{array}{c}
0 \\
0 \\
0 \\
\Gamma_{\mathrm{e} 4} \\
\Gamma_{e 5}
\end{array}\right]+\left[\begin{array}{c}
J_{1}^{T} \\
J_{2}^{T} \\
0 \\
0 \\
0
\end{array}\right]=\left[\begin{array}{ccccc}
M_{11} & M_{12} & 0 & 0 & 0 \\
M_{21} & M_{22} & 0 & M_{23} & M_{24} \\
0 & 0 & 0 & 0 & 0 \\
0 & M_{32} & 0 & M_{33} & 0 \\
0 & M_{42} & 0 & 0 & M_{44}
\end{array}\right]\left[\begin{array}{c}
\ddot{h}_{1} \\
\ddot{h}_{2} \\
0 \\
\ddot{h}_{3} \\
\ddot{h}_{4}
\end{array}\right]+
$$

$\left[\begin{array}{lllcc}0 & C_{12} & 0 & 0 & 0 \\ 0 & C_{22} & 0 & C_{23} & C_{24} \\ 0 & 0 & 0 & 0 & 0 \\ 0 & C_{32} & 0 & 0 & C_{34} \\ 0 & C_{42} & 0 & C_{43} & 0\end{array}\right]\left[\begin{array}{c}\dot{h}_{1} \\ \dot{h}_{2} \\ 0 \\ \dot{h}_{3} \\ \dot{h}_{4}\end{array}\right]+\left[\begin{array}{c}V_{1} \\ V_{2} \\ 0 \\ 0 \\ 0\end{array}\right]+\left[\begin{array}{c}\eta_{1} \\ \eta_{2} \\ 0 \\ \eta_{4} \\ \eta_{5}\end{array}\right]$

The model is described by four equations which correspond respectively to the frame translations, frame rotations, the steering and the wheels rotations.

From this new representation, we estimate the forces translations as following:

$F_{T}=J_{1}^{T} F=M_{11} \ddot{h}_{1}+M_{12} \ddot{h}_{2}+C_{12} \dot{h}_{2}+V_{1}+\eta_{1}$

The next equation describes the forces rotations of the frame.

$F_{R}=J_{2}^{T} F=M_{21} \ddot{h}_{1}+M_{22} \ddot{h}_{2}+C_{22} \dot{h}_{2}+C_{23} \dot{h}_{3}+C_{24} \dot{h}_{4}+$

$V_{2}+\eta_{2}$

The forces of steering and wheels rotations are:
$\Gamma_{e 4}=M_{32} \ddot{h}_{2}+M_{33} \ddot{h}_{3}+C_{32} \dot{h}_{2}+C_{34} \dot{h}_{4}+V_{3}+\eta_{4}$

$\Gamma_{e 5}=M_{42} \ddot{h}_{2}+M_{44} \ddot{h}_{4}+C_{42} \dot{h}_{2}+C_{43} \dot{h}_{3}+V_{4}+\eta_{5}$

The spitting of the 12 DoF model can be written as:

$\sum 11: \ddot{h}_{1}=f_{1}\left(h_{1}, \dot{h}_{1}, F_{T}\right)+\eta_{c}^{1}$

$\sum 12: \ddot{h}_{2}=f_{2}\left(h_{2}, \dot{h}_{2}, F_{R}\right)+\eta_{c}^{2}$

$\sum 31: \ddot{h}_{3}=f_{3}\left(h_{3}, \dot{h}_{3}, \Gamma_{e 3}\right)+\eta_{c}^{4}$

$\sum 32: \ddot{h}_{4}=f_{4}\left(h_{4}, \dot{h}_{4}, \Gamma_{e 4}\right)+\eta_{c}^{5}$

\section{C.1 Representation in 2 under systems:}

Movements of the frame: The equation (c18) gives a general frame representation (translation and rotation): $\sum 1$ :

$\left[\begin{array}{l}F_{T} \\ F_{R}\end{array}\right]=\left[\begin{array}{ll}M_{11} & M_{12} \\ M_{21} & M_{22}\end{array}\right]\left[\begin{array}{l}\ddot{h}_{1} \\ \ddot{h}_{2}\end{array}\right]+\left[\begin{array}{l}C_{12} \\ C_{22}\end{array}\right] \dot{h}_{2}+\left[\begin{array}{l}V_{1} \\ V_{2}\end{array}\right]+\left[\begin{array}{l}\eta_{c}^{1} \\ \eta_{c}^{2}\end{array}\right]$

The two coupling terms $\eta_{c}^{1}$ and $\eta_{c}^{2}$ verify:

$\eta_{c}^{1}=\eta_{1}$

$\eta_{c}^{2}=M_{23} \ddot{h}_{3}+M_{24} \ddot{h}_{4}+C_{23} \dot{h}_{3}+C_{24} \dot{h}_{4}+\eta_{2}$

By choosing the state variables $x_{11}=\left(h_{1}, h_{2}\right)$ and $x_{12}=$ $\left(\dot{h}_{1}, \dot{h}_{2}\right)$. We have the following state representation:

$\left\{\begin{aligned} \dot{x}_{11} & =x_{12} \\ \dot{x}_{12} & =M_{1}^{-1}\left(J_{12}^{T} F-C_{1} x_{12}-V_{12}-\mu_{1}\right) \\ y_{1} & =s\left(x_{11}, x_{12}\right)\end{aligned}\right.$

With: $M_{1}=\left[\begin{array}{ll}M_{11} & M_{12} \\ M_{21} & M_{22}\end{array}\right], \quad C_{1}=\left[\begin{array}{l}C_{12} \\ C_{22}\end{array}\right], \quad J_{12}^{T}=\left[\begin{array}{l}J_{1}^{T} \\ J_{2}^{T}\end{array}\right], \quad V_{12}=$ $\left[\begin{array}{l}V_{1} \\ V_{2}\end{array}\right]$ and $\mu_{1}=\left[\begin{array}{l}\eta_{c}^{1} \\ \eta_{c}^{2}\end{array}\right]$ Which are respectively the inertia matrix, the matrix of centrifugal and Coriolis forces, the reduced Jacobian matrix, vector gravity and the vector of coupling terms linked to the first sub system.

Dynamic of wheels:

The wheels dynamic $\left(\sum 2\right)$ contains two blocks: the steering of the two front wheels $\left(\sum 31\right)$ and the rotations of four wheels following their axis $\mathrm{y}\left(\sum 32\right)$.

The eq(c12, c13) give the following dynamic model:

$\Gamma_{e 3}=M_{33} \ddot{h}_{3}+C_{34} \dot{h}_{4}+\eta_{c}^{4}$

$\Gamma_{e 4}=M_{44} \ddot{h}_{4}+C_{43} \dot{h}_{3}+\eta_{c}^{5}$

By identification, we obtain the coupling terms $\eta_{c}^{4}$ and $\eta_{c}^{5}$ :

$\eta_{c}^{4}=M_{32} \ddot{h}_{2}+C_{32} \dot{h}_{2}+\eta_{4}$

$\eta_{c}^{5}=M_{42} \ddot{h}_{2}+C_{42} \dot{h}_{2}+\eta_{5}$

If we combine the equations together we will have:

$\left[\begin{array}{l}\Gamma_{e 4} \\ \Gamma_{e 5}\end{array}\right]=\left[\begin{array}{cc}M_{33} & 0 \\ 0 & M_{44}\end{array}\right]\left[\begin{array}{l}\ddot{q}_{3} \\ \ddot{q}_{4}\end{array}\right]+\left[\begin{array}{lr}0 & C_{34} \\ C_{43} & 0\end{array}\right]\left[\begin{array}{l}\dot{q}_{3} \\ \dot{q}_{4}\end{array}\right]+\left[\begin{array}{l}\eta_{c}^{4} \\ \eta_{c}^{5}\end{array}\right]$

For the same reasoning, we chose the state variables $x_{21}=\left(h_{3}, h_{4}\right)$ and $x_{22}=\left(\dot{h}_{3}, \dot{h}_{4}\right)$, in order to obtain the following state representation:

$\left\{\begin{array}{l}\dot{x}_{21}=x_{22} \\ \dot{x}_{22}=M_{2}^{-1}\left(\Gamma_{e 45}-C_{2} x_{22}-\mu_{2}\right) \\ y_{2}=s\left(x_{21}, x_{22}\right)\end{array}\right.$

With : $M_{2}=\left[\begin{array}{cc}M_{33} & 0 \\ 0 & M_{44}\end{array}\right], C_{2}=\left[\begin{array}{lr}0 & C_{34} \\ C_{43} & 0\end{array}\right], \Gamma_{e 45}=\left[\begin{array}{l}\Gamma_{e 4} \\ \Gamma_{e 5}\end{array}\right]$, and $\mu_{3}=\left[\begin{array}{l}\eta_{c}^{4} \\ \eta_{c}^{5}\end{array}\right]$ Which are respectively the reduced inertia matrix, the reduced matrix of centrifugal and Coriolis forces, the vector of input torque and the vector of coupling terms attached to the second sub system. In conclusion the main difference with previous models is that there is no suspension dynamics (one line and one column disappear from inertial matrix and Coriolis and centrifuge matrix. The coupling terms are simpler as consequence.

\section{Fourth application for a motorcycle:}


A motorcycle is a single-track, two-wheeled motor vehicle. Different types of motorcycles have different dynamics and these play a role in how a motorcycle performs in given conditions.

We split its dynamic model while considering: the inertia matrix $(5 \times 5)$ and the state vector are expressed as following sets: $h^{T}=\left[h_{1}^{T} h_{2}^{T} h_{3}^{T} h_{4}^{T} h_{5}^{T}\right]$

With: $h_{1}^{T}=\left[q_{1} q_{2} q_{3}\right] h_{2}^{T}=\left[q_{4} q_{5} q_{6}\right], h_{3}^{T}=\left[q_{7} q_{8}\right]$

$h_{4}^{T}=\left[q_{9}\right] h_{5}^{T}=\left[\begin{array}{ll}q_{10} & q_{11}\end{array}\right]$

In the same way, we subdivide vectors $\Gamma_{e}, V(q, \dot{q})$ and $\eta_{0}(t, q, \dot{q})$ as it follows:

$\Gamma_{e}=\left[0,0,0, \Gamma_{e 4}, \Gamma_{e 5}\right]^{\mathrm{T}}$

$V(q, \dot{q})=\left[\begin{array}{lll}V_{1} V_{2} V_{3} & 0 & 0\end{array}\right]^{T}$

$\eta_{0}(t, q, \dot{q})=\left[\eta_{1} \eta_{2} \eta_{3} \eta_{4} \eta_{5}\right]^{T}$

Then the 11 DoF model of the motorcycle has the following form:

$\left[\begin{array}{c}0 \\ 0 \\ 0 \\ \Gamma_{\mathrm{e} 4} \\ \Gamma_{e 5}\end{array}\right]+\left[\begin{array}{c}J_{1}^{T} \\ J_{2}^{T} \\ J_{3}^{T} \\ 0 \\ 0\end{array}\right] F=\left[\begin{array}{ccccc}M_{11} & M_{12} & M_{13} & 0 & 0 \\ M_{21} & M_{22} & M_{23} & M_{24} & M_{25} \\ M_{31} & M_{32} & M_{33} & 0 & 0 \\ 0 & M_{42} & M_{43} & M_{44} & 0 \\ 0 & M_{52} & 0 & 0 & M_{55}\end{array}\right]\left[\begin{array}{c}\ddot{h}_{1} \\ \ddot{h}_{2} \\ \ddot{h}_{3} \\ \ddot{h}_{4} \\ \ddot{h}_{5}\end{array}\right]+$

$\left[\begin{array}{ccccc}0 & C_{12} & C_{13} & 0 & 0 \\ 0 & C_{22} & C_{23} & C_{24} & C_{25} \\ 0 & C_{32} & 0 & 0 & 0 \\ 0 & C_{42} & 0 & 0 & C_{45} \\ 0 & C_{52} & 0 & C_{54} & 0\end{array}\right]\left[\begin{array}{c}\dot{h}_{1} \\ \dot{h}_{2} \\ \dot{h}_{3} \\ \dot{h}_{4} \\ \dot{h}_{5}\end{array}\right]+\left[\begin{array}{c}V_{1} \\ V_{2} \\ V_{3} \\ 0 \\ 0\end{array}\right]+\left[\begin{array}{l}\eta_{1} \\ \eta_{2} \\ \eta_{3} \\ \eta_{4} \\ \eta_{5}\end{array}\right]$

The model is described by five equations which correspond respectively to the frame translations, frame rotations, suspension system, the steering and the wheels rotations. Basing on equation (d9), we constate that the form is similar to the P406 model.

\section{E. Fifth application for a bicycle:}

Bicycle, also known as bike, is a pedal-driven, having two wheels attached to frame which one behind the other. We considered a bike without any suspension system and we subdivide bicycle dynamic model while considering: the inertia matrix and the state vector are expressed as following: $h^{T}=\left[h_{1}^{T} h_{2}^{T} 0 h_{3}^{T} h_{4}^{T}\right]$

With: $h_{1}^{T}=\left[q_{1} q_{2} q_{3}\right] h_{2}^{T}=\left[q_{4} q_{5} q_{6}\right], h_{3}^{T}=\left[q_{7}\right]$

$h_{4}^{T}=\left[q_{8} q_{9}\right]$

In the same way, we subdivide vectors $\Gamma_{e}, V(q, \dot{q})$ and $\eta_{0}(t, q, \dot{q})$ as it follows:

$\Gamma_{e}=\left[0,0,0, \Gamma_{e 3}, \Gamma_{e 4}\right]^{\mathrm{T}}$

$V(q, \dot{q})=\left[\begin{array}{llll}V_{1} V_{2} & 0 & 0 & 0\end{array}\right]^{T}$

$\eta_{0}(t, q, \dot{q})=\left[\begin{array}{lll}\eta_{1} \eta_{2} 0 & \eta_{3} \eta_{4}\end{array}\right]^{T}$

The model is described by four equations which correspond respectively to the frame translations, frame rotations, the steering and the wheels rotations.

Then the 9dof model of the bicycle has the following form:

$\left[\begin{array}{c}0 \\ 0 \\ 0 \\ \Gamma_{\mathrm{e} 3} \\ \Gamma_{e 4}\end{array}\right]+\left[\begin{array}{c}J_{1}^{T} \\ J_{2}^{T} \\ 0 \\ 0 \\ 0\end{array}\right]=\left[\begin{array}{ccccc}M_{11} & M_{12} & 0 & 0 & 0 \\ M_{21} & M_{22} & 0 & M_{23} & M_{24} \\ 0 & 0 & 0 & 0 & 0 \\ 0 & M_{32} & 0 & M_{33} & 0 \\ 0 & M_{42} & 0 & 0 & M_{44}\end{array}\right]\left[\begin{array}{c}\ddot{h}_{1} \\ \ddot{h}_{2} \\ 0 \\ \ddot{h}_{3} \\ \ddot{h}_{4}\end{array}\right]+$

$\left[\begin{array}{lllcc}0 & C_{12} & 0 & 0 & 0 \\ 0 & C_{22} & 0 & C_{23} & C_{24} \\ 0 & 0 & 0 & 0 & 0 \\ 0 & C_{32} & 0 & 0 & C_{34} \\ 0 & C_{42} & 0 & C_{43} & 0\end{array}\right]\left[\begin{array}{c}\dot{h}_{1} \\ \dot{h}_{2} \\ 0 \\ \dot{h}_{3} \\ \dot{h}_{4}\end{array}\right]+\left[\begin{array}{c}V_{1} \\ V_{2} \\ 0 \\ 0 \\ 0\end{array}\right]+\left[\begin{array}{c}\eta_{1} \\ \eta_{2} \\ 0 \\ \eta_{3} \\ \eta_{4}\end{array}\right]$
In this case, we note that forces and coupling terms have similar form as the electrical kart (see P3.3).

\section{F. Interpretation:}

To summarize, the following figure 6 describes the five blocks of the global and the second subset (the frame translations, frame rotations, suspension system, the steering and the wheels rotations).

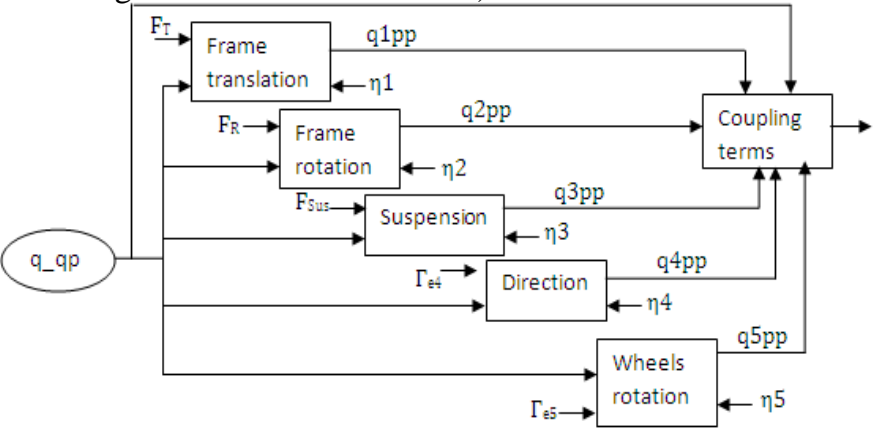

Fig.6: the 5 blocks of the P406, Quad and motorcycle

The following figure 7 describes the four blocks of the third subset (the frame translations, frame rotations, the steering and the wheels rotations) with no suspension system.

The 4 blocks of kart and bike dynamic models acquire the same properties as the global system (see paragraph 2). Thus it is important to remark that the coupling terms and the uncertainties of modeling, estimation and noises are going to appear in the same way as passive inputs in the system. So they will be dissipated.

It will be the same for other sub systems of the nominal model, because the same reasoning applies "mutatis mutandis". The importance of our approach shows that the employement of the neglected terms are well chosen.

In this paper we consider the car as a parent agent and quad, kart, motorcycle and bicycle as its descendants. In fact, the reference dynamic model is the 16 DoF model which we will identify blocks of each descendant in this global model to facilitate any task switching from one system to another or from one structure to another. The table 2 shows a correlation between the kart and a P406 models as follows:

- The dashed blocks represent the dynamics of the kart;

- The not dashed blocks represent the dynamics provided by the four suspensions of P406.

With this strategy we can classify the five models using only the global car model for ambitions goals such as drawing, motion analysis and diagnosis...

\section{SIMULATION V.}

The dynamic model of the kart without contact is generated with the symbolic calculations software (Maple).

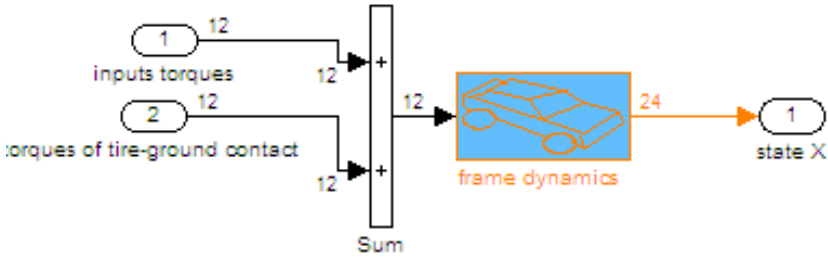

Figure 8: Block of simulation under MATLAB 
To validate this decoupled model, detailed in the previous paragraph, we used a modified simulator version of (SimK106N) (G. Beurier [8] M'sirdi [12]). For this simulation, we apply a constant direction $-0.7^{\circ}$;

So we will take the kart model for the following simulation as an example to analyze the evolution of coupling terms compared with torques.

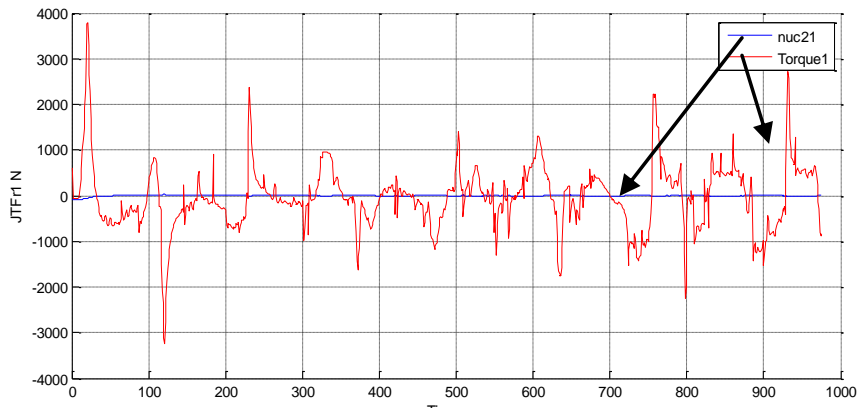

Figure 9: The evolution of $2^{\text {Timg ms }}$ coupling term

We compared the terms of the equations c21. We observe that the amplitudes of coupling terms are lower than the input torque (fig. 9). The absence of a suspension system on the kart is translated by vibration of these parameters. On a normal car the coupling terms $\eta_{c}^{i}$ are passive and converge to zero.

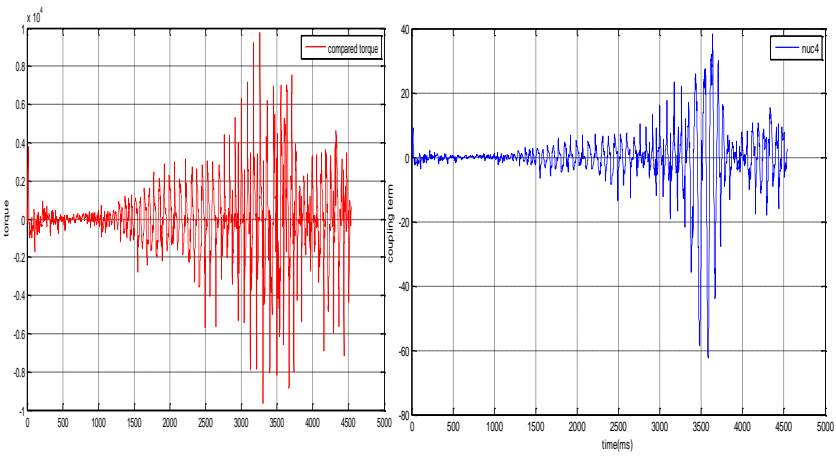

Figure 10.a: The evolution of compared torque Figure 10.b: The evolution of $4^{\text {rd }}$ coupling term

Even, we can neglect the $4{ }^{\text {rd }}$ coupling term to the compared torques (see Fig. 10 and equation c27). On the other side, the excellent attenuation of $\eta_{c}^{i}$ in the case of a car is explained by the absorption of these parameters at the suspension system level.

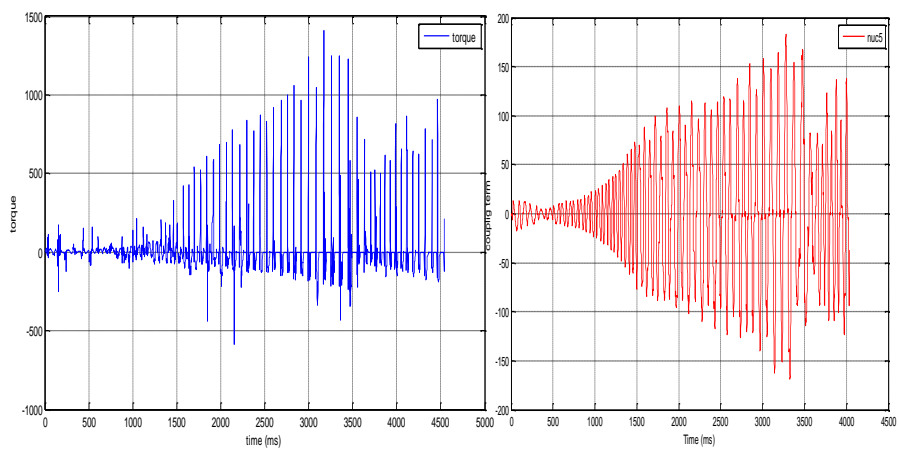

Figure 11.a: The evolution of steering torque term Figure 11.b: The evolution of $5^{\text {th }}$ coupling term
Using equation c27, we obtain figure 11 which shows that the $5^{\text {th }}$ coupling term is smaller than the amplitudes of the torque of wheels rotations and it can be neglected. Also the equation (c19) proves that the $1^{\text {st }}$ coupling term is equal to a neglected term $\eta_{1}$ because of the absence of a suspension system.

\section{CONCLUSION}

In this paper, a 16 DoF global dynamic model of a car is developed and exploited with its suspension system. This geometrical and kinematics model takes into account the unknown tire-ground contact forces. For diagnosis purpose based on decentralized robust observers, we split the model in sub-blocks in order to illustrate the passivity of the global system and the sub systems. We have also compared dynamics of 5 vehicles. This comparison allows understanding the rationale of the behavior of vehicles in perspectives this will be used for control design and diagnosis. The simulator (SimK106N) was transformed to simulate the dynamic model of five kind of vehicles, allowed us to appreciate the coupling energy level flowing through the whole systems.

\section{REFERENCES}

[1] N.K. M'sirdi, A. Rabhi, N. Zbiri, and Y. Delanne: VRIM: Vehicle Road Interaction Modelling for Estimation of Contact Forces. Accepted for TMVDA 04. 3rd Int. Tyre Colloquium Tyre Models For Vehicle Dynamics Analysis August 30-31, 2004 University of Technology Vienna. [2] N.K. M'Sirdi, A. Rabhi, Aziz Naamane: Vehicle models and estimation of contact forces and tire road friction". Invited paper ICINCO: 351-358, 2007.

[3] H.B. Pacejka.: Simplified behavior of steady state turning behavior of motor vehicles, part 1: Handling diagrams and simple systems. Vehicle System Dynamics 2 1973, pp. 162 - 172.

[4] B. Jaballah- NK M'sirdi - A Naamane, M. Hassani "Estimation of Longitudinal and Lateral Velocity of Vehicle" , "17th IEEE Mediterranean Conference on Control and Automation (MED'09).

[5] A Rabhi, NK M'sirdi A Naamane, B Jaballah. Vehicle Velocity Estimation Using Sliding Mode Observers" , in : IEEE, "9th int conf Sciences and Techniques of Automatic, STA'2008

[6] K. N. M'sirdi, L. H. Rajaoarisoa, J.-F. Balmat, J. Duplaix: Modeling for Control and diagnosis for a class of Non Linear complex switched systems. Advances in Vehicle Control and Safety AVCS 07, Buenos Aires, Argentine, February 8-10, 2007.

[7] Khalil w. "Dynamic modelling and identification of a car" 2002 CIFA.

[8] G. Beurier Thèse de doctorat de l'UPMC, Paris 6.

[9] Khalil w. Kleinfinger J-F "minimum operations and minimum parameters of the dynamic model of tree structure robots" IEEE of Robotics and Automation, déc. 1987

[10] N.K. M'sirdi, B. Jaballah, A. Naamane, H. Messaoud: Robust Observers and Unknown Input Observers for estimation, diagnosis and control of vehicle dynamics, IEEE/RSJ International Conference on IROS.

[11] H Nasser, N.K. M'Sirdi, , A Naamane "Inboard observers and sensors for an electrical and autonomous kart" STA 2008.

[12] N. K. M'Sirdi. «Observateurs robustes et estimateurs pour l'estimation de la dynamique des véhicules et du contact pneu - route ». JAA. Bordeaux, 5-6 Nov 2003. 